\title{
The Boltzmann-Hamel Equations for Optimal
}

\section{Control}

\author{
Jared M. Maruskin and Anthony M. Bloch \\ Department of Mathematics, The University of Michigan
}

\begin{abstract}
We will extend the Boltzmann-Hamel equations to the optimal control setting, producing a set of equations for both kinematic and dynamic nonholonomic optimal control problems. In particular, we will show the dynamic optimal control problem can be written as a minimal set of $4 n-2 m$ first order differential equations of motion.
\end{abstract}

Key words:

Nonholonomic Control, Optimal Control, Boltzmann-Hamel equations, quasi-velocities

\section{INTRODUCTION}

\subsection{Overview}

Quasi-velocity formulations, such as Maggi's equation and the BoltzmannHamel equation, have achieved much success in the analysis of nonholonomic systems due to their ability to cast the dynamical equations of motion in a 
form requiring fewer equations, see $(10),(13)$, and $(11)$. For an $n$ degree of freedom system with $m$ nonholonomic constraints, $2 n+m$ equations of motion are required if one uses the fundamental nonholonomic form of Lagranges equation. $2 n$ differential equations for the system state, and $m$ algebraic relations that must be solved for the multipliers. However, if quasi-velocity techniques are employed, the system can be written as a system of $2 n-m$ first order differential equations.

The standard approach to optimal control problems is to use Lagrange Multipliers. Under certain conditions, the optimal control problem can be reformulated as a vakonomic (variational nonholonomic) problem (3) . One can further analyze optimal control problems with Pontryagin's Maximum Principle, see (2), (5), or (1). Solutions to the kinematic optimal control problems, where one has direct control over a number of the velocities, can be expressed using $2 n+m$ equations of motion; whereas solutions to dynamical optimal control problems, where one has acceleration controls, can be expressed with $4 n+m$ equations of motion. Some geometric aspects of this system have been discussed in (6). In this paper, we extend quasi-velocity techniques to optimal control problems with nonholonomic constraints. We show how to write the optimal control equations for kinematically actuated systems as a system of $2 n$ first order differential equations (a savings of $m$ equations) and the optimal control equations for dynamically actuated systems as a system of $4 n-2 m$ first order differential equations (a savings of $3 m$ equations). 


\subsection{Summation Convention}

To aid in notation, we will invoke the summation convention throughout this paper. Greek letters $(\alpha, \beta, \gamma, \ldots)$ run over the constrained dimensions $1, \ldots, m$. Capital letters $(A, B, C, \ldots)$ run over the unconstrained dimensions $m+1, \ldots, n$. Lower case letters $(a, b, c, \ldots)$ run over all dimensions $1, \ldots, n$.

\section{QUASI-VELOCITIES AND VARIATIONS}

In this section we will present the basic background on nonholonomic constraints and quasi-velocities. We will discuss the basic properties of this connection and derive the transpositional relations, (10), (13).

\subsection{Nonholonomic Constraints and Quasi-Velocities}

Let $Q$ be the configuration manifold of our system, with $\operatorname{dim} Q=n$ and $T Q$ its corresponding tangent bundle (our phase space). A mechanical Lagrangian is

given by $L: T Q \rightarrow \mathbb{R}$, usually taken to have the form $L(q, \dot{q})=g_{i j} \dot{q}^{i} \dot{q}^{j}-V(q)$ where $g_{i j}$ is the kinetic energy metric and $V(q)$ is a potential term.

We further suppose our system is subject to $m$ linear scleronomic (time independent) nonholonomic constraints, i.e. constraints of the form:

$$
a_{i}^{\alpha}(q) \dot{q}^{i}=0
$$

Define now a vector space isomorphism $\Psi_{i}^{j}$ on the tangent space, with inverse transformation $\Phi_{j}^{i}$. The first $m$ rows of $\Psi_{i}^{j}$ are taken to agree with the constraint matrix, i.e. $\Psi_{i}^{\sigma}(q)=a_{i}^{\sigma}(1)$. The remaining rows can be choosen freely, 
so long as the resulting matrix $\Psi$ is invertible. The transformation $\Psi$ can be viewed as a change of basis:

$$
\Psi:\left\{\frac{\partial}{\partial q^{i}}\right\}_{i=1}^{n} \rightarrow\left\{\frac{\partial}{\partial \theta^{i}}\right\}_{i=1}^{n}
$$

where the new basis is referred to as the quasi-basis. The velocity of the system $v \in T_{q} Q$ can be expressed in terms of the ordinary or quasi-basis as follows:

$$
\dot{q}^{i} \frac{\partial}{\partial q^{i}}=\left(\Psi_{i}^{j} \dot{q}^{i}\right) \frac{\partial}{\partial \theta^{j}}=u^{j} \frac{\partial}{\partial \theta^{j}}=\left(\Phi_{j}^{i} u^{j}\right) \frac{\partial}{\partial q^{i}}
$$

where the components $u^{j}$ are the quasi-velocities. Basis vectors transform as:

$$
\frac{\partial}{\partial \theta^{j}}=\Phi_{j}^{i} \frac{\partial}{\partial q^{i}} \quad \text { and } \quad \frac{\partial}{\partial q^{i}}=\Psi_{j}^{i} \frac{\partial}{\partial \theta^{j}}
$$

Finally, one defines a set of $n$ one-forms, dual to the quasi-basis:

$$
d \theta^{j}=\Psi_{i}^{j} d q^{i}
$$

Even though this notation is found in the literature, it is really a notational misnomer, as the one forms $d \theta^{j}$ are not exact.

\subsection{Variations}

Definition 1 Consider a curve $\gamma(t):[a, b] \rightarrow Q$. A proper variation of $\gamma(t)$ is a differentiable function $q(s, t):[-\varepsilon, \varepsilon] \times[a, b] \rightarrow Q$ that satisfies the following conditions:

(i) $q(0, t)=\gamma(t), \quad \forall t \in[a, b]$

(ii) $q(s, a)=\gamma(a)$ and $q(s, b)=\gamma(b), \forall s \in[-\varepsilon, \varepsilon]$.

Definition 2 The infinitessimal variation $\delta q(t)$ corresponding to the variation $q(s, t)$ is the vector field defined along $\gamma(t)$ by $\delta q(t)=\left.\frac{\partial q(s, t)}{\partial s}\right|_{s=0}$. 
We will further assume the variations to be continuous and contemporaneous. Continuity of the variations implies that the Lie Derivative $\mathcal{L}_{\dot{q}} \delta q \equiv 0$ vanishes identically. Contemporaneous variations occur without the passage of time. The infinitessimal variations, when expressed in terms of the quasi-basis, are given by $\delta \theta^{j}(t)=\Psi_{i}^{j} \delta q^{i}$.

\subsection{The Transpositional Relations}

A fundamental ingredient for understanding nonholonomic variational problems is the following set of transpositional relations (see (10), (13)).

Theorem 1 (First Transpositional Relations) Utilizing the shorthand $d:=$ $\partial / \partial t, \delta:=\partial / \partial s$, we have:

$$
\left(d \delta q^{i}-\delta d q^{i}\right) \Psi_{i}^{j}=\left(d \delta \theta^{j}-\delta d \theta^{j}\right)+\gamma_{a b}^{j} u^{a} \delta \theta^{b}
$$

where $\gamma_{a b}^{j}$ are the Hamel coefficients $\gamma_{p q}^{s}=\left\{\frac{\partial \Psi_{i}^{s}}{\partial q^{j}}-\frac{\partial \Psi_{j}^{s}}{\partial q^{i}}\right\} \Phi_{p}^{i} \Phi_{q}^{j}$

The left hand side of $(2)$ is no more than $d \theta^{j}\left(\mathcal{L}_{\dot{q}} \delta q\right)$; and, therefore, for continuous variations, is identically zero. We therefore have the following:

Corollary 1 For proper, continuous variations, variations of the quasi-velocities can be related to variations of the quasi-coordinates as follows:

$$
\delta u^{j}=d \delta \theta^{j}+\gamma_{a b}^{j} u^{a} \delta \theta^{b}
$$

Therefore, due to the nonintegrability of the constraint distribution $\left(\gamma_{i j}^{\sigma} \neq 0\right.$, $\sigma=1, \ldots, m)$, one cannot obtain closure in the quasi-coordinate space, even at the differential level $\left((10)\right.$, (13) ). One must choose between $\delta u^{\sigma}=0$ or $d \delta \theta^{j}=$ 0. The correct dynamical equations of motion are obtained if one chooses the 
variations so that they obey the Principle of Virtual Work, $\delta \theta^{j} \equiv 0$. If one, on the other hand, choose the variations to satisfy $\delta u^{\sigma}=0$, one would obtain trajectories that satisfy Hamilton's Principle. Such trajectories are referred to as the vakonomic motion of the system, a term introduced by Arnold.

Definition 3 The associated quasi-acceleration, $a^{i}$, and quasi-jerk, $\jmath^{i}$, are defined to be $a^{i}=\dot{u}^{i} \quad$ and $\quad j^{i}=\dot{a}^{i}$.

A direct coordinate calculation shows:

Theorem 2 (Second Transpositional Relation) For continuous variations, we have $\delta d u^{i}=d \delta u^{i}$. Equivalently, $\delta a^{i}=\partial\left(\delta u^{i}\right) / \partial t$.

\section{THE BOLTZMANN-HAMEL EQUATIONS}

We will derive the Boltzmann-Hamel equations for nonholonomic mechanics directly from variational principles. A more algebraic derivation of these equations is given in (10). We will begin with the Lagrange-D'Alembert Principle:

Definition 4 (Lagrange-D'Alembert Principle) The correct dynamical equations of motion are the ones which minimize the action $I=\int_{a}^{b} L(q, \dot{q}) d t$, where $L(q, \dot{q})$ is the unconstrained mechanical Lagrangian and the variations are chosen to satisfy the Principle of Virtual Work.

Let $\mathscr{L}(q, u)=L(q, \dot{q}(q, u))$ be the re-expression of the unconstrained Lagrangian in terms of the quasi-velocities. Taking variations of the action and using the first transpositional relations (3), one obtains: 


$$
\begin{aligned}
\delta I & =\int_{a}^{b}\left(\frac{\partial \mathscr{L}}{\partial q^{i}} \delta q^{i}+\frac{\partial \mathscr{L}}{\partial u^{i}} \delta u^{i}+F_{i} \delta q^{i}\right) d t \\
& =\int_{a}^{b}\left(\frac{\partial \mathscr{L}}{\partial \theta^{i}}-\frac{d}{d t} \frac{\partial \mathscr{L}}{\partial u^{i}}+\frac{\partial \mathscr{L}}{\partial u^{j}} \gamma_{k i}^{j} u^{k}+Q_{i}\right) \delta \theta^{i} d t
\end{aligned}
$$

where $F_{i}$ is the external applied force and we have defined:

$$
\frac{\partial \mathscr{L}}{\partial \theta^{i}}=\frac{\partial \mathscr{L}}{\partial q^{j}} \frac{\partial q^{j}}{\partial \theta^{i}}=\frac{\partial \mathscr{L}}{\partial q^{j}} \Phi_{i}^{j} \quad \text { and } \quad Q_{i}=\Phi_{i}^{j} F_{j}
$$

After applying the Principle of Virtual Work, $\delta \theta^{\sigma} \equiv 0$, the remaining $n-m$ variations $\delta \theta^{I}$ can be taken to be independent, and we obtain the BoltzmannHamel equations for nonholonomic mechanics:

$$
\begin{aligned}
\frac{d}{d t} \frac{\partial \mathscr{L}}{\partial u^{I}}-\frac{\partial \mathscr{L}}{\partial \theta^{I}}-\frac{\partial \mathscr{L}}{\partial u^{j}} \gamma_{K I}^{j} u^{K} & =Q_{I} \\
\dot{q}^{i} & =\Phi_{J}^{i} u^{J}
\end{aligned}
$$

One must use the unconstrained Lagrangian for these equations. After the partial derivatives are taken, one then applies the constraints $u^{\sigma}=0$. The Boltzmann-Hamel equations (4)- (5) are a minimal set of $2 n-m$ first order differential equations for the $n q^{i}$ 's and the $n-m u^{I}$ 's.

\section{KINEMATIC OPTIMAL CONTROL}

In this section we will present a quasi-velocity based method for kinematic optimal control problems, where one has direct controls over the velocities. As an example, we will work out the optimal kinematic control equations for the falling rolling disc. 


\subsection{Theory}

For a general affine kinematic control system subject to $m$ nonholonomic constraints, the following system is typically specified: $\dot{q}^{i}=X_{I}^{i}(q) w^{I}$, where the $w^{I}$ are the $n-m$ controls and $X_{I}^{i}(q)$ is the $i$-th component of the $I$-th independent control vector field. Taking the $m$ constraints as the first $m$ quasi-velocities:

$$
u^{\sigma}=\Psi_{i}^{\sigma} \dot{q}^{i} \equiv 0
$$

one can, wlog, take the controls as the remaining independent quasi-velocities:

$$
w^{I}(q, \dot{q})=u^{I}=\Psi_{i}^{I} \dot{q}^{i}
$$

With this choice, the control vector fields are thus identifies with the last $n-m$ columns of $\Phi=\Psi^{-1}$, i.e. $X_{I}^{i}=\Phi_{I}^{i}$.

For a given cost integrand $g(q, w)$, the Kinematic Optimal Control Problem

is then given by minimizing the cost function $I=\int_{a}^{b} g(q, w) d t$ over all curves satisfying (7)-(6) with fixed endpoints $q(a)$ and $q(b)$.

We now define the quasi-basis so that $\Psi_{i}^{\sigma}=a_{i}^{\sigma}$, as usual, and, additionally, so that $\Psi_{i}^{I}=b_{i}^{I}$. Then the constraints can be written $u^{\sigma}$, and the $n-m$ control variables $w^{I}$ coincide with the remaining $n-m$ free quasi-velocities $u^{I}$. Define now $C(q, u)=g(q, w(q, \dot{q}(q, u)))$. In our case, we have chosen the unconstrained quasi-velocities to coincide with the controls, i.e. $u^{I}=w^{I}$, thus we will have $C(q, u)=g(q, u)$.

In order to enforce (6), we must apply the Lagrange Multipliers to the cost function before taking variations. In this case, we are selecting Hamilton's Principle, where the cost function is minimized amongst the set of kinematically 
admissable curves. We then take unconstrained variations of the augmented cost function $I=\int_{a}^{b}\left(C(q, u)+\mu_{\sigma} u^{\sigma}\right) d t$. Since $C(q, u)$ only depends on the unconstrained quasi-velocities $u^{I}$, we have:

$$
\delta I=\int_{a}^{b}\left(\frac{\partial C}{\partial \theta^{i}} \delta \theta^{i}+\frac{\partial C}{\partial u^{I}} \delta u^{I}+\mu_{\sigma} \delta u^{\sigma}+u^{\sigma} \delta \mu_{\sigma}\right) d t
$$

Setting the coefficients of the $\delta \mu_{\sigma}$ terms returns our constraints $u^{\sigma}=0$. Leaving this term off for now, using the transpositional relations (3), and integrating by parts yields

$$
\delta I=\int_{a}^{b}\left\{\left(\frac{\partial C}{\partial \theta^{i}}+\frac{\partial C}{\partial u^{I}} \gamma_{s i}^{I} u^{s}+\mu_{\sigma} \gamma_{s i}^{\sigma} u^{s}\right) \delta \theta^{i}-\frac{d}{d t} \frac{\partial C}{\partial u^{I}} \delta \theta^{I}-\dot{\mu}_{\sigma} \delta \theta^{\sigma}\right\} d t
$$

We thus have the following

Theorem 3 The Boltzmann-Hamel equations for the kinematic optimal control problem are:

$$
\begin{aligned}
\frac{d}{d t} \frac{\partial C}{\partial u^{I}}-\frac{\partial C}{\partial \theta^{I}}-\frac{\partial C}{\partial u^{J}} \gamma_{S I}^{J} u^{S} & =\mu_{\tau} \gamma_{S I}^{\tau} u^{S} \\
-\frac{\partial C}{\partial \theta^{\sigma}}-\frac{\partial C}{\partial u^{J}} \gamma_{S \sigma}^{J} u^{S} & =-\dot{\mu}_{\sigma}+\mu_{\tau} \gamma_{S \sigma}^{\tau} u^{S} \\
\dot{q}^{i} & =\Phi_{S}^{i} u^{S}
\end{aligned}
$$

These represent a minimal set of $2 n$ first order differential equations: the $n-m$ equations (8) for the unconstrained $u^{I}$ 's, the $m$ equations (9) for the multipliers $\mu_{\sigma}$ 's, and $n$ kinematic relations (10) for the $q^{i}$ 's.

As an interesting aside, if the cost function integrand $C(q, u)$, when expressed in terms of the quasi-velocities, is identical to the constrained mechanical Lagrangian, then these equations produce the vakonomic motion associated with the system. See (3) for additional discussion on the coincidence of the vakonomic motion (Lagrange's Problem) and the optimal control problem. 


\subsection{Optimal Control of the Heisenberg System}

The optimal control of the Heisenberg system, discussed in (4) and (2), is a classical underactuated kinematic control problem. Local coordinates are given by $q=\langle x, y, z\rangle$. For this system, one has velocity controls $w_{1}=\dot{x}$ and $w_{2}=\dot{y}$ and the motion is subject to the nonholonomic constraint $\dot{z}=y \dot{x}-x \dot{y}$. The control velocity field is therefore given by:

$$
\dot{q}=X_{1} w^{1}+X_{2} w^{2}
$$

where $X_{1}=\langle 1,0, y\rangle^{T}$ and $X_{2}=\langle 0,1,-x\rangle^{T}$. Using these controls, one seeks to steer the particle from the point $\langle 0,0,0\rangle$ at time $t=0$ to the point $\langle 0,0, a\rangle$ at time $T>0$, while minimizing the functional $I=\frac{1}{2} \int_{0}^{T}\left(w_{1}^{2}+w_{2}^{2}\right) d t$.

We will derive the equations of motion which yield this solution path via the vakonomic form of the Boltzmann-Hamel equations. We choose quasivelocities: $u_{1}=y \dot{x}-x \dot{y}-\dot{z}, \quad u_{2}=\dot{x}$, and $u_{3}=\dot{y}$. Notice the quasi-velocities $u_{2}$ and $u_{3}$ coincide with the control velocities. The transformation matrices $\Psi$ and $\Phi$ are given by:

$$
\Psi=\left[\begin{array}{ccc}
y & -x & -1 \\
1 & 0 & 0 \\
0 & 1 & 0
\end{array}\right] \text { and } \Phi=\left[\begin{array}{ccc}
0 & 1 & 0 \\
0 & 0 & 1 \\
-1 & y & -x
\end{array}\right]
$$

The nonzero Hamel coefficients are $\gamma_{23}^{1}=-\gamma_{32}^{1}=2$. Expressing the integrand of the cost function in terms of quasi-velocities yields $C=\frac{1}{2}\left(u_{2}^{2}+u_{3}^{2}\right)$. The kinematic optimal control Boltzmann-Hamel equations (8)-(10) immediately produce the following set of first order differential equations:

$$
\begin{array}{ccl}
\dot{x}=u_{2} & \dot{y}=u_{3} & \dot{z}=-u_{2}+y u_{2}-x u_{3} \\
\dot{u}_{2}=-2 \mu u_{3} & \dot{u}_{3}=2 \mu u_{2} & \dot{\mu}=0
\end{array}
$$


where $\mu(t)=\mu(0)$ is an arbitrary constant that can be choosen such that the solution trajectory reaches its final destination point. The top equations are a reiteration of the control field $\dot{q}=X_{1} w^{1}+X_{2} w^{2}=X_{1} u^{2}+X_{2} u^{3}$ and the bottom equations produce the optimal control.

\subsection{Optimal Control of the Vertical Rolling Disc}

The generalized coordinates of the vertical rolling disc are given by $q=$ $\langle x, y, \theta, \phi\rangle$, where $(x, y)$ is the contact point of the disc and the $x-y$ plane, $\phi$ is the angle the disc makes with the $x$-axis, and $\phi$ is the angle a reference point on the disc makes with the vertical. Assume we have the kinematic controls $w_{1}=\dot{\theta}$ and $w_{2}=\dot{\phi}$, and that the motion is subject to the nonholonomic constraints $\dot{x}-\cos (\phi) \dot{\theta}=0$ and $\dot{y}-\sin (\phi) \dot{\theta}=0$. This gives rise to the control vector field $\dot{q}=X_{1} w^{1}+X_{2} w^{2}$ where $X_{1}=\langle\cos \phi, \sin \phi, 1,0\rangle^{T}$ and $X_{2}=\langle 0,0,0,1\rangle^{T}$

We wish to steer the disc between two points while minimizing the cost functional $\frac{1}{2} \int_{a}^{b}\left(w_{1}^{2}+w_{2}^{2}\right) d t$. We choose quasi-velocities $u_{1}=\dot{x}-\cos (\phi) \dot{\theta}$, $u_{2}=\dot{y}-\sin (\phi) \dot{\theta}, u_{3}=\dot{\theta}$, and $u_{4}=\dot{\phi}$, so that the transformation matrices $\Psi$ and $\Phi$ are given by:

$$
\Psi=\left[\begin{array}{cccc}
1 & 0 & -\cos \phi & 0 \\
0 & 1 & -\sin \phi & 0 \\
0 & 0 & 1 & 0 \\
0 & 0 & 0 & 1
\end{array}\right] \Phi=\left[\begin{array}{cccc}
1 & 0 & \cos \phi & 0 \\
0 & 1 & \sin \phi & 0 \\
0 & 0 & 1 & 0 \\
0 & 0 & 0 & 1
\end{array}\right]
$$

The Hamel coefficients are: $\gamma_{34}^{1}=\sin \phi=-\gamma_{43}^{1}$ and $\gamma_{34}^{2}=-\cos \phi=-\gamma_{43}^{2}$. In terms of the quasi-velocities, the integrand of the cost function becomes $C(q, u)=\frac{1}{2} u_{3}^{2}+\frac{1}{2} u_{4}^{2}$. The Boltzmann-Hamel equations (8)-(10) then produce the following set of first order differential equations: 


$$
\begin{array}{llll}
\dot{u}_{3}=\left(\mu_{2} \cos \phi-\mu_{1} \sin \phi\right) u_{4} & \dot{\mu}_{1}=0 & \dot{x}=\cos (\phi) u_{3} & \dot{\theta}=u_{3} \\
\dot{u}_{4}=\left(\mu_{1} \sin \phi-\mu_{2} \cos \phi\right) u_{3} & \dot{\mu}_{2}=0 & \dot{y}=\sin (\phi) u_{3} & \dot{\phi}=u_{4}
\end{array}
$$

\subsection{Kinematic Optimal Control of the Falling Rolling Disc}

The falling rolling disc can be described by the contact point $(x, y)$ and Classical Euler angles $(\phi, \theta, \psi)$, as shown in Figure 1. We will take the coordinate ordering $(\phi, \theta, \psi, x, y)$.

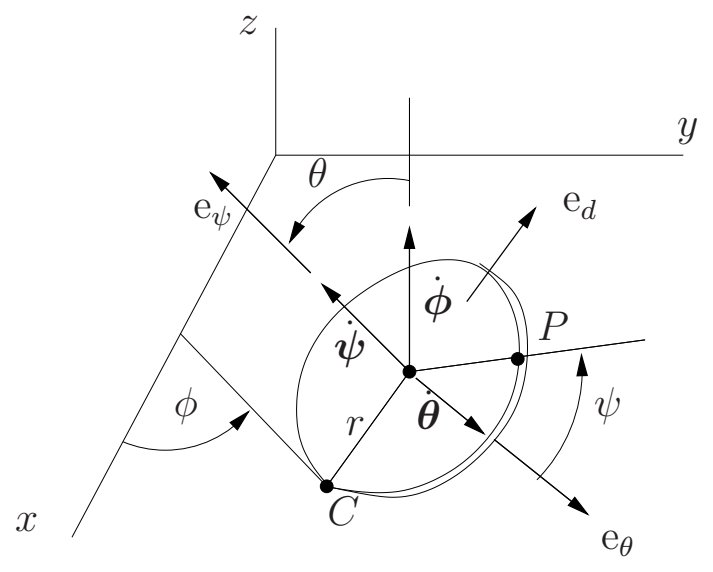

Fig. 1. Euler Angles of the Falling Rolling Disc

Suppose we have direct control over the body-axis angular velocities $w_{1}=$ $\omega_{d}:=\dot{\phi} \sin \theta, w_{2}=\dot{\theta}$, and $w_{3}=\Omega:=\dot{\phi} \cos \theta+\dot{\psi}$ (in the $\mathrm{e}_{d}, \mathrm{e}_{\theta}$, and $\mathrm{e}_{\psi}$ directions, respectively (see Fig. 1)), and the system is subject to the nonholonomic constraints $\dot{x}+r \dot{\psi} \cos \phi=0$ and $\dot{y}+r \dot{\psi} \sin \phi=0$. We wish to steer the disc between two points while minimizing the cost functional $I[\gamma]=\frac{1}{2} \int_{a}^{b}\left(w_{1}^{2}+w_{2}^{2}+w_{3}^{2}\right) d t$. We will choose as quasi-velocities $u_{1}=\dot{\phi} \sin \theta$, $u_{2}=\dot{\theta}, u_{3}=\dot{\phi} \cos \theta+\dot{\psi}, u_{4}=\dot{x}+r \dot{\psi} \cos \phi$, and $u_{5}=\dot{y}+r \dot{\psi} \sin \phi$. The quasivelocities $\left(u_{1}, u_{2}, u_{3}\right)=\left(\omega_{d}, \dot{\theta}, \Omega\right)$ represent the angular velocity expressed in the body-fixed frame, and are coincident with the kinematic controls. These are not true velocities (like the Euler Angle Rates), as they are non-integrable. The nonholonomic constraints in terms of these variables are $u_{4}=u_{5}=0$. 
The transformation matrices are

$$
\Psi=\left[\begin{array}{ccccc}
\sin \theta & 0 & 0 & 0 & 0 \\
0 & 1 & 0 & 0 & 0 \\
\cos \theta & 0 & 1 & 0 & 0 \\
0 & 0 & r \cos \phi & 1 & 0 \\
0 & 0 & r \sin \phi & 0 & 1
\end{array}\right] \quad \text { and } \quad \Phi=\left[\begin{array}{ccccc}
\csc \theta & 0 & 0 & 0 & 0 \\
0 & 1 & 0 & 0 & 0 \\
-\cot \theta & 0 & 1 & 0 & 0 \\
r \cos \phi \cot \theta & 0 & -r \cos \phi & 1 & 0 \\
r \sin \phi \cot \theta & 0 & -r \sin \phi & 0 & 1
\end{array}\right]
$$

The nonzero Hamel-coefficients are $\gamma_{21}^{1}=-\cot \theta=-\gamma_{12}^{1}, \gamma_{21}^{3}=1=-\gamma_{12}^{3}$, $\gamma_{13}^{4}=r \sin \phi \csc \theta=-\gamma_{31}^{4}$, and $\gamma_{13}^{5}=-r \cos \phi \csc \theta=-\gamma_{31}^{5}$.

Written in terms of the quasi-velocities, the integrand of the cost function is $C(q, u)=\frac{1}{2}\left(u_{1}^{2}+u_{2}^{2}+u_{3}^{2}\right)$. The kinematic optimal control Boltzmann-Hamel equations (8)-(10) give us a minimal set of 10 first order differential equations:

$$
\begin{aligned}
\dot{u}_{1} & =u_{2} u_{3}-u_{1} u_{2} \cot \theta-r\left(\mu_{4} \sin \phi-\mu_{5} \cos \phi\right) \csc \theta u_{3} \\
\dot{u}_{2} & =u_{1}^{2} \cot \theta-u_{1} u_{3} \\
\dot{u}_{3} & =r\left(\mu_{4} \sin \phi-\mu_{5} \cos \phi\right) \csc \theta u_{1} \\
\dot{\mu}_{4} & =0, \quad \dot{\mu}_{5}=0 \\
\dot{\phi} & =\csc \theta u_{1}, \quad \dot{\theta}=u_{2}, \quad \dot{\psi}=-\cot \theta u_{1}+u_{3} \\
\dot{x} & =r \cos \phi \cos \theta u_{1}-r \cos \phi u_{3}, \quad \dot{y}=r \sin \phi \cot \theta u_{1}-r \sin \phi u_{3}
\end{aligned}
$$

\section{DYNAMIC OPTIMAL CONTROL}

In this section, we will derive a set of Boltzmann-Hamel equations for the dynamic optimal control problem, which is normally a fourth order system. We will present a minimal set of $4 n-2 m$ first order differential equations that produces the optimal control, and then discuss examples. 


\subsection{Boltzmann-Hamel Equations for Optimal Dynamic Control}

Given a nonholonomic mechanical system with $n-m$ independent acceleration controls, it can be recast into the form given by the dynamical BoltzmannHamel equations (4)-(5). The dynamical optimal control problem is the problem of finding solution curves between two fixed points $\langle q(a), \dot{q}(a)\rangle$ and $\langle q(b), \dot{q}(b)\rangle$

that minimize the cost function $I=\int_{a}^{b} g(q, \dot{q}, Q) d t$. Utilizing (4) and (5), we can rewrite the integrand as an explicit function of the coordinates, quasivelocities, and quasi-accelerations $C(q, u, a)=g(q, \dot{q}(q, u), Q(q, u, a))$.

Since the Boltzmann-Hamel equations no longer depend on the constrained quasi-velocities and quasi-accelerations, $C(q, u, a)$ is also independent of $u^{\sigma}$ and $a^{\sigma}$. Taking variations yields: $\delta I=\int\left\{\frac{\partial C}{\partial q^{i}} \delta q^{i}+\frac{\partial C}{\partial u^{J}} \delta u^{J}+\frac{\partial C}{\partial a^{J}} \delta a^{J}\right\} d t$. Using the second transpositional relations Theorem 2 for $\delta a^{J}$ and then integrating by parts we obtain $\delta I=\int\left\{\frac{\partial C}{\partial q^{i}} \delta q^{i}+\left[\frac{\partial C}{\partial u^{J}}-\frac{d}{d t} \frac{\partial C}{\partial a^{J}}\right] \delta u^{J}\right\} d t$. Defining the parameters

$$
\kappa_{J}=\frac{\partial C}{\partial u^{J}}-\frac{d}{d t} \frac{\partial C}{\partial a^{J}}
$$

and using the first Transpositional relations (3) we obtain:

$$
\delta I=\int\left\{\frac{\partial C}{\partial \theta^{r}}-\dot{\kappa}_{J} \delta_{r}^{J}+\kappa_{J} \gamma_{s r}^{J} u^{s}\right\} \delta \theta^{r} d t
$$

These variations are not free, but subject to the nonholonomic constraints $a_{i}^{\sigma} \dot{q}^{i}=0$. We form the augmented cost integrand by replacing $C(q, u, a)$ with $C(q, u, a)+\mu_{\sigma} u^{\sigma}$. Taking variations, the $\delta \mu^{\sigma}$ coefficients recover the constraints. Ignoring these terms, we are left with $\delta I=$

$$
\int\left\{\frac{\partial C}{\partial \theta^{r}}-\dot{\kappa}_{J} \delta_{r}^{J}+\kappa_{J} \gamma_{s r}^{J} u^{s}-\dot{\mu}_{\sigma} \delta_{r}^{\sigma}+\mu_{\sigma} \gamma_{s r}^{\sigma} u^{s}\right\} \delta \theta^{r} d t
$$

where the variations are now taken to be unconstrained. Notice the multipliers 
$\mu_{\sigma}$ are not the mechanical multipliers, but a multiplier on the cost function that enforces Hamilton's Principle. We thus have the following:

Theorem 4 The Boltzmann-Hamel equations for Optimal Dynamic Control are given by:

$$
\begin{aligned}
-\frac{\partial C}{\partial \theta^{A}}+\dot{\kappa}_{A}-\kappa_{J} \gamma_{S A}^{J} u^{S} & =\mu_{\tau} \gamma_{S A}^{\tau} u^{S} \\
-\frac{\partial C}{\partial \theta^{\sigma}}-\kappa_{J} \gamma_{S \sigma}^{J} u^{S} & =\mu_{\tau} \gamma_{S \sigma}^{\tau} u^{S}-\dot{\mu}_{\sigma} \\
\dot{q}^{i} & =\Phi_{S}^{i} u^{S}
\end{aligned}
$$

The optimal control system can therefore be given by a minimal set of $4 n-2 m$ first order differential equations as follows. We have $n$ kinematic relations (14), $2 n-2 m$ relations $\dot{u}^{A}=a^{A}$ and $\dot{a}^{A}=\jmath^{A}, n-m$ equations for $j^{A}$ (given by inserting (11) into (12)), and, finally, $m$ relations for the multipliers $\dot{\mu}_{\sigma}(13)$. Once the resulting optimal control dynamics are determined, the control forces which produce the optimal trajectory are then given by the $n-m$ algebraic equations (4). The solution is then found by solving the related boundary value problem, with $4 n-2 m$ prescribed boundary conditions: $q^{i}(0), u^{A}(0), q^{i}(T), u^{A}(T)$.

\subsection{Dynamic Optimal Control of the Vertical Rolling Disc}

Consider the vertical rolling disc of $\S 4.3$ with control torques in the $\theta$ and $\phi$ directions. The corresponding dynamical equations of motion (see (2)) are: $\frac{3}{2} \ddot{\theta}=w_{3}, \frac{1}{4} \ddot{\phi}=w_{4}, \dot{x}=\dot{\theta} \cos \phi$, and $\dot{y}=\dot{\theta} \sin \phi$. This is equivalent to a minimal set of 6 first order differential equations (the number obtained by using the Boltzmann-Hamel equations (4) and (5). 
We now wish to choose the control forces so as to minimize the cost function $\int \frac{1}{2}\left(w_{3}^{2}+w_{4}^{2}\right) d t$. Solving for the controls in terms of the quasi-accelerations $w_{3}=\frac{3}{2} \ddot{\theta}=\frac{3}{2} a_{3}$ and $w_{4}=\frac{1}{4} \ddot{\phi}=\frac{1}{4} \ddot{a}_{4}$, this is equivalent to minimizing the action $\int\left(\frac{9}{8} a_{3}^{2}+\frac{1}{32} a_{4}^{2}\right) d t$ subject to the nonholonomic constraints. Using the dynamic optimal control Boltzmann-Hamel equations (12) and (13), coupled with the dynamical equations of motion above, and eliminating the controls, we have a minimal system of 12 first order differential equations:

$$
\begin{array}{rlrl}
\dot{x}=\cos \phi & u_{3} & j_{3} & =\frac{4}{9}\left(\mu_{1} \sin \phi-\mu_{2} \cos \phi\right) u_{4} \\
\dot{y}=\sin \phi u_{3} & j_{4} & =16\left(-\mu_{1} \sin \phi+\mu_{2} \cos \phi\right) u_{3} \\
\dot{\theta}=u_{3} \dot{u}_{3}=a_{3} \dot{a}_{3} & =\jmath_{3} & \dot{\mu}_{1}=0 \\
\dot{\phi}=u_{4} \dot{u}_{4}=a_{4} \dot{a}_{4} & =\jmath_{4} & \dot{\mu}_{2}=0
\end{array}
$$

By use of quasi-velocities, quasi-accelerations, and quasi-jerks, we have made the following simplifications: $u_{1}=u_{2}=a_{1}=a_{2}=\jmath_{1}=\jmath_{2}=0$, thereby eliminating the necessity of 6 of the 18 first order differential equations necessary in the standard approach. The solution to this system of differential equations yields the optimal dynamic control equations of the vertical rolling disc. It is equivalent to the following reduced system

$$
\begin{aligned}
\dot{x}=\cos \phi \dot{\theta} & \dot{y}=\sin \phi \dot{\theta} \\
\dddot{\theta}=\frac{4}{9}\left(\mu_{1} \sin \phi-\mu_{2} \cos \phi\right) \dot{\phi} & \dddot{\phi}=16\left(-\mu_{1} \sin \phi+\mu_{2} \cos \phi\right) \dot{\theta}
\end{aligned}
$$

where $\mu_{1}, \mu_{2}$ are constants.

\subsection{Dynamic Optimal Control of the Free Rigid Body}

Consider dynamic control of the free rigid body, where the generalized coordinates are given by the Type-I Euler angles $(\psi, \theta, \phi)$. As quasi-velocities, choose 
the body-fixed components of the angular momentum $u_{1}=\omega_{x}=-\dot{\psi} \sin \theta+\dot{\phi}$, $u_{2}=\omega_{y}=\dot{\psi} \cos \theta \sin \phi+\dot{\theta} \cos \phi$, and $u_{3}=\omega_{z}=\dot{\psi} \cos \theta \cos \phi-\dot{\theta} \sin \phi$. The transformation matrices are given as:

$$
\Psi=\left[\begin{array}{ccc}
-\sin \theta & 0 & 1 \\
\cos \theta \sin \phi & \cos \phi & 0 \\
\cos \theta \cos \phi & -\sin \phi & 0
\end{array}\right] \quad \text { and } \quad \Phi=\left[\begin{array}{ccc}
0 & \sec \theta \sin \phi & \sec \theta \cos \phi \\
0 & \cos \phi & -\sin \phi \\
1 & \tan \theta \sin \phi & \tan \theta \cos \phi
\end{array}\right]
$$

The mechanical Lagrangian is given as $\mathscr{L}(q, u)=\frac{1}{2}\left(I_{x x} u_{1}^{2}+I_{y y} u_{2}^{2}+I_{z z} u_{3}^{2}\right)$. The nonzero Hamel coefficients are $\gamma_{23}^{1}=1, \gamma_{13}^{2}=-1, \gamma_{12}^{3}=1, \gamma_{32}^{1}=-1$, $\gamma_{31}^{2}=1$, and $\gamma_{21}^{3}=-1$. For notational convenience, define $\eta_{32}=I_{z z}-I_{y y}$, $\eta_{13}=I_{x x}-I_{z z}$, and $\eta_{21}=I_{y y}-I_{x x}$. Then the Boltzmann-Hamel equations (4) produce the Euler Equations:

$$
I_{x x} \dot{u}_{1}+\eta_{32} u_{2} u_{3}=M_{x} \quad I_{y y} \dot{u}_{2}+\eta_{13} u_{1} u_{3}=M_{y} \quad I_{z z} \dot{u}_{3}+\eta_{21} u_{1} u_{2}=M_{z}
$$

where $M_{x}, M_{y}$, and $M_{z}$ are the control torques applied about the body fixed principal axes. The cost function integrand $\frac{1}{2}\left(M_{x}^{2}+M_{y}^{2}+M_{z}^{2}\right)$, when expressed in terms of quasi-variables, is given by: $C=\frac{1}{2}\left\{I_{x x}^{2} a_{1}^{2}+I_{y y} a_{2}^{2}+I_{z z} a_{3}^{2}+\right.$ $\left.2 I_{x x} \eta_{32} a_{1} u_{2} u_{3}+2 I_{y y} \eta_{13} u_{1} a_{2} u_{3}+2 I_{z z} \eta_{21} u_{1} u_{2} a_{3}+\eta_{32}^{2} u_{2}^{2} u_{3}^{2}+\eta_{13}^{2} u_{1}^{2} u_{3}^{2}+\eta_{21}^{2} u_{1}^{2} u_{2}^{2}\right\}$.

The $\kappa$ 's (11) are given by:

$$
\begin{aligned}
\kappa_{1}= & I_{y y} \eta_{13} a_{2} u_{3}+I_{z z} \eta_{21} u_{2} a_{3}+\eta_{13}^{2} u_{1} u_{3}^{2}+\eta_{21}^{2} u_{1} u_{2}^{2} \\
& -I_{x x} J_{1}-I_{x x} \eta_{32} u_{2} a_{3}-I_{x x} \eta_{32} a_{2} u_{3} \\
\kappa_{2}= & I_{x x} \eta_{32} a_{1} u_{3}+I_{z z} \eta_{21} u_{1} a_{3}+\eta_{32}^{2} u_{2} u_{3}^{2}+\eta_{21}^{2} u_{1}^{2} u_{2} \\
& -I_{y y} J_{2}-\eta_{13} I_{y y} u_{1} a_{3}-\eta_{13} I_{y y} a_{1} u_{3} \\
\kappa_{3}= & I_{x x} \eta_{32} a_{1} u_{2}+I_{y y} \eta_{13} u_{1} a_{2}+\eta_{32}^{2} u_{2}^{2} u_{3}+\eta_{13}^{2} u_{1}^{2} u_{3} \\
& -I_{z z} J_{3}-\eta_{21} I_{z z} u_{1} a_{2}-\eta_{21} I_{z z} a_{1} u_{2}
\end{aligned}
$$

The optimal control Boltzmann-Hamel equations (12) then work out to be:

$$
\dot{\kappa}=\kappa \times \omega
$$

These provide 3 differential equations for the $j$ 's. Let $\mathbb{I}$ be the moment inertia 
tensor with respect to the principal axes basis $\hat{\mathbf{e}}_{x}, \hat{\mathbf{e}}_{y}$, $\hat{\mathbf{e}}_{z}$, so that, in dyadic notation, $\mathbb{I}=I_{x x} \hat{\mathbf{e}}_{x} \hat{\mathbf{e}}_{x}+I_{y y} \hat{\mathbf{e}}_{y} \hat{\mathbf{e}}_{y}+I_{z z} \hat{\mathbf{e}}_{z} \hat{\mathbf{e}}_{z}$. Let $\boldsymbol{\Pi}:=\mathbb{I} \cdot \boldsymbol{\omega}$ be the body axis angular momentum, and $\boldsymbol{\kappa}=\left\langle\kappa_{1}, \kappa_{2}, \kappa_{3}\right\rangle$. Then (16)-(18) can alternatively be re-expressed as:

$$
\kappa=\Pi \times \dot{\Pi}+\Pi \times(\omega \times \Pi)-\ddot{\Pi}-\mathbb{I} \cdot\{2 \boldsymbol{\omega} \times \dot{\Pi}+\dot{\boldsymbol{\omega}} \times \Pi+\boldsymbol{\omega} \times(\boldsymbol{\omega} \times \Pi)\}
$$

Finally, by defining $\boldsymbol{\lambda}(\boldsymbol{\omega}, \dot{\boldsymbol{\omega}})=\boldsymbol{\kappa}+\ddot{\boldsymbol{\Pi}}$, the dynamic optimal control equations for the free rigid body can be expressed as:

$$
\dddot{\Pi}=\dot{\lambda}+\ddot{\Pi} \times \omega-\lambda \times \omega
$$

In addition, we have the kinematic relations

$$
\begin{aligned}
\dot{\psi} & =\sec \theta \sin \phi u_{2}+\sec \theta \cos \phi u_{3} \\
\dot{\theta} & =\cos \phi u_{2}-\sin \phi u_{3} \\
\dot{\phi} & =u_{1}+\tan \theta \sin \phi u_{2}+\tan \theta \cos \phi u_{3}
\end{aligned}
$$

as well as the relations $\dot{u}_{i}=a_{i}, \dot{a}_{i}=\jmath_{i}$. This is a set of 12 first order differential equations. Once one solves the corresponding boundary value problem (initial, final Euler angles, angular velocities specified), the controls are determins by the algebraic relations (15).

For the special case when the rigid body is spherical one sees from (20) that $\boldsymbol{\kappa}=-\ddot{\boldsymbol{\Pi}}$ and $\boldsymbol{\lambda}=\mathbf{0}$. Then the Boltzmann-Hamel equations for the optimal dynamic control of the free rigid body (21) reduce to $\ddot{\boldsymbol{\omega}}=\ddot{\boldsymbol{\omega}} \times \boldsymbol{\omega}$. When coupled with the kinematic relations (22)-(24) and the algebraic relations (15), the optimal control trajectories of the free rigid sphere are produced. Integrating once yields the second order system $\ddot{\omega}=\mathbf{c}+\dot{\boldsymbol{\omega}} \times \boldsymbol{\omega}$, which coincides with the result of (12). See also (8). The optimal solution trajectory of the reorientation of the rigid sphere from $\mathbf{q}(0)=\langle 0,0,0\rangle, \boldsymbol{\omega}(0)=\langle 0,0,0\rangle$ to the point $\mathbf{q}(1)=$ 

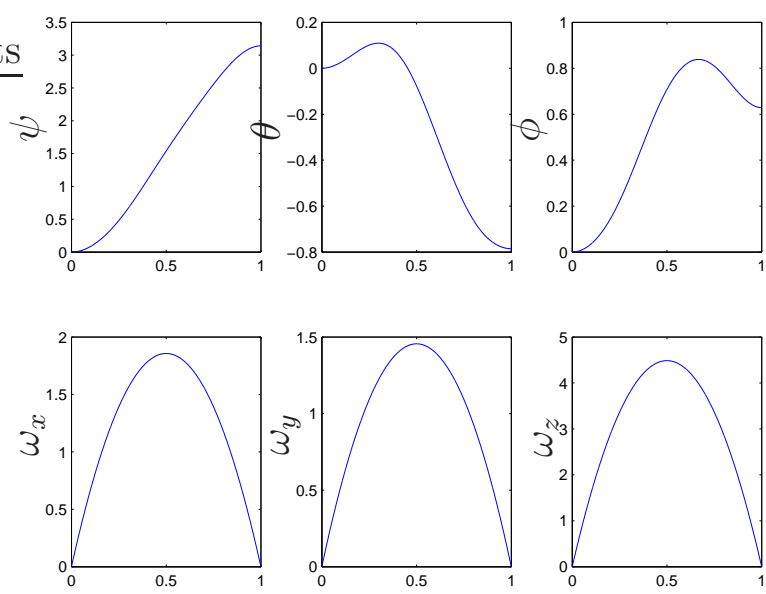

Fig. 2. Optimal Dynamic Control of Free Sphere: Euler Angles and Body Fixed Angular Velocity with respect to time.

$\langle\pi,-\pi / 4, \pi / 5\rangle, \boldsymbol{\omega}(1)=\langle 0,0,0\rangle$ is plotted in Fig. 2 .

\section{CONCLUSIONS}

In this paper, we showed how one can extend quasi-velocity techniques to kinematic and optimal control problems. Standard Lagrange Multiplier techniques for kinematical optimal control problems produce a set of $2 n+m$ first order differential equations: $n$ for the coordinates $q^{i}, n$ for the velocities $\dot{q}^{i}$, and $m$ for the multipliers $\mu_{\sigma}$. On the other hand, by generalizing the dynamic Boltzmann-Hamel equations to the kinematic control setting (Theorem 3), we obtain a savings of $m$ first order differential equations, as one no longer need solve for the constrained quasi-velocities. Moreover, the differential equations for the multipliers (9) are naturally separated from the differential equations for the quasi-velocities (8).

For the dynamic optimal control problem, one typically encounters a fourth order system, plus multipliers, which produces a total of $4 n+m$ first order 
differential equations. The Boltzmann-Hamel form of the equations (Theorem 4) gives a minimal set of $4 n-2 m$ equations of motion, as one no longer need integrate the $m$ constrained quasi-velocities, quasi-accelerations, and quasi-

jerks, $u^{\sigma} \equiv 0, a^{\sigma} \equiv 0, \jmath^{\sigma} \equiv 0$, respectively. This approach gives us a total savings of $3 m$ first order differential equations. Initial and final conditions are then enforced by solving the resulting system of differential equations as a two point boundary value problem.

The authors wish to thank support from NSF grants DMS-0604307 and CMS0408542 .

\section{References}

[1] A. Agrachev and Y. Sachkov, Control Theory from the Geometric Viewpoint, Springer, 2004.

[2] A.M. Bloch, Nonholonomic Mechanics and Control, Springer, 2003.

[3] A.M. Bloch and P.E. Crouch, "Reduction of Euler Lagrange Problems for Constrained Variational Problems and Relation with Optimal Control Problems", In The Proceedings of the 33rd IEEE Conference on Decision and Control: 2584 - 2590, 1994.

[4] R.W. Brockett, "Control Theory and Singular Riemannian Geometry", in New Directions in Applied Mathematics, Springer-Verlag, 11-27, 1981.

[5] F. Bullo and A.D. Lewis, Geometric Control of Mechanical Systems, Springer, 2005.

[6] M. Camarinha, F. Silva Leite, and P. Crouch, "On the Geometry of Riemannian Cubic Polynomials", Differential Geometry and its Applications, 15: 107-135, 2001.

[7] F. Cardin and M. Favretti, "On Nonholonomic and Vakonomic Dynamics of Mechanical Systems with Nonintegrable Constraints", Journal of Geometry and Physics, 18 (4): 295-325, 1996.

[8] P. Crouch and F. Silva Leite, "The Dynamic Interpolation Problem: On Riemannian Manifolds, Lie Groups, and Symmetric Spaces", Journal of Dynamical and Control Systems, 1 (2): 177-202, 1995.

[9] M. Favretti, "Equivalence of Dynamics for Nonholonomic Systems with Transverse Constraints," Journal of Dynamics and Differential Equations, 10 (4): 511-535, 1998.

[10] D.T. Greenwood, Advanced Dynamics, Cambridge University Press, 2003.

[11] J.I. Neimark and N.A. Fufaev, Dynamics of Nonholonomic Systems, Translations of Mathematical Monographs, AMS, 33.

[12] L. Noakes, G. Heinzinger, and B. Paden, "Cubic Splines on Curved Spaces", IMA Journal of Mathematical Control and Information, 6: 465-473, 1989.

[13] J.G. Papastavridis, Analytical Mechanics, Oxford University Press, 2002. 